\title{
Parallel-sequencing of early-type and spiral galaxies
}

\author{
Michele Cappellari \\ Sub-department of Astrophysics, Department of Physics, University of Oxford \\ Denys Wilkinson Building, Keble Road, Oxford OX1 3RH \\ email: cappellari@astro.ox.ac.uk
}

\begin{abstract}
Since Edwin Hubble introduced his famous tuning fork diagram more than 70 years ago, spiral galaxies and early-type galaxies (ETGs) have been regarded as two distinct families. The spirals are characterized by the presence of disks of stars and gas in rapid rotation, while the early-types are gas poor and described as spheroidal systems, with less rotation and often non-axisymmetric shapes. The separation is physically relevant as it implies a distinct path of formation for the two classes of objects. I will give an overview of recent findings, from independent teams, that motivated a radical revision to Hubble's classic view of ETGs. These results imply a much closer link between spiral galaxies and ETGs than generally assumed.
\end{abstract}

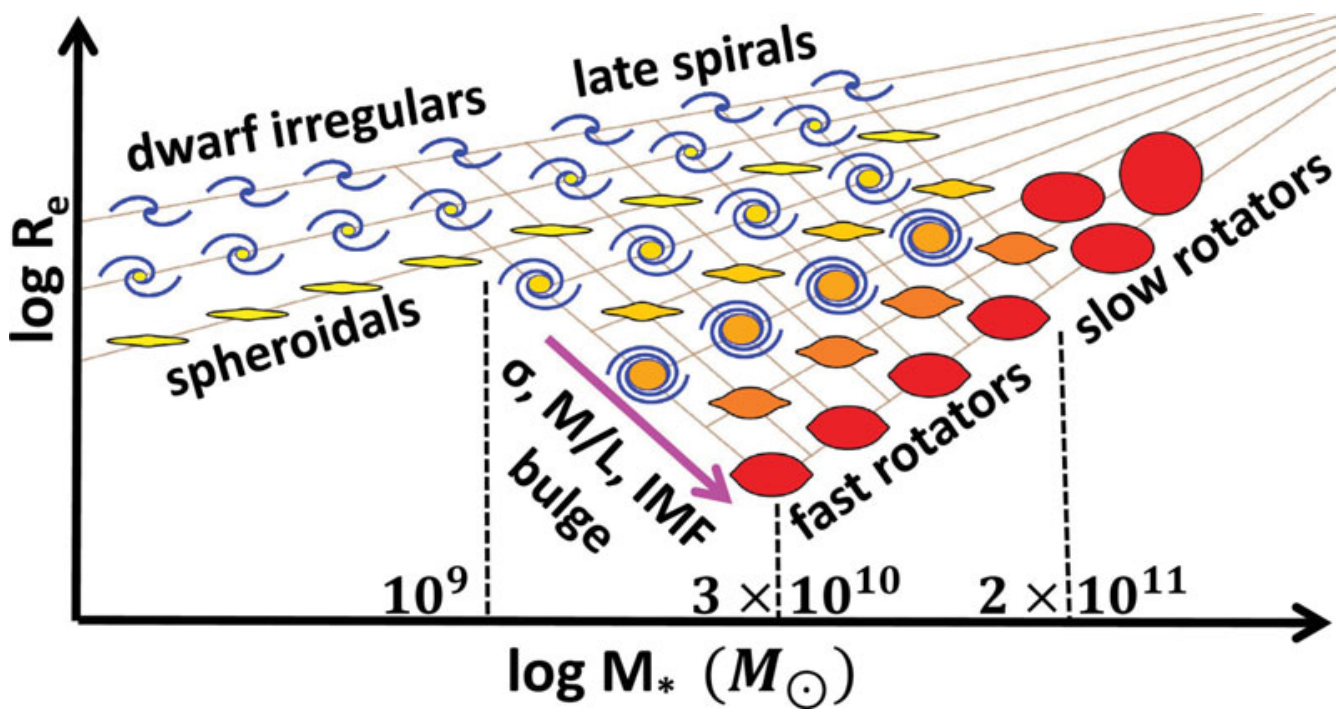

Figure 1. ETGs properties like shape, dynamics, population and IMF, merge smoothly with the properties of spiral galaxies on the mass-size diagram. All trends appear driven by an increase of the bulge fraction, which greatly enhance the likelihood for a galaxy to have his star formation quenched. This parallelism between the properties of spirals and ETGs motivated a proposed revision of Hubble's tuning-fork diagram. The same symbols are used in this figure (taken from Cappellari et al. 2012) as in the 'comb' morphological classification diagram proposed in Cappellari et al. (2011).

\section{References}

Cappellari, M., et al., 2011, MNRAS, 416, 1680

Cappellari, M., et al., 2013, MNRAS, 432, 1862 